
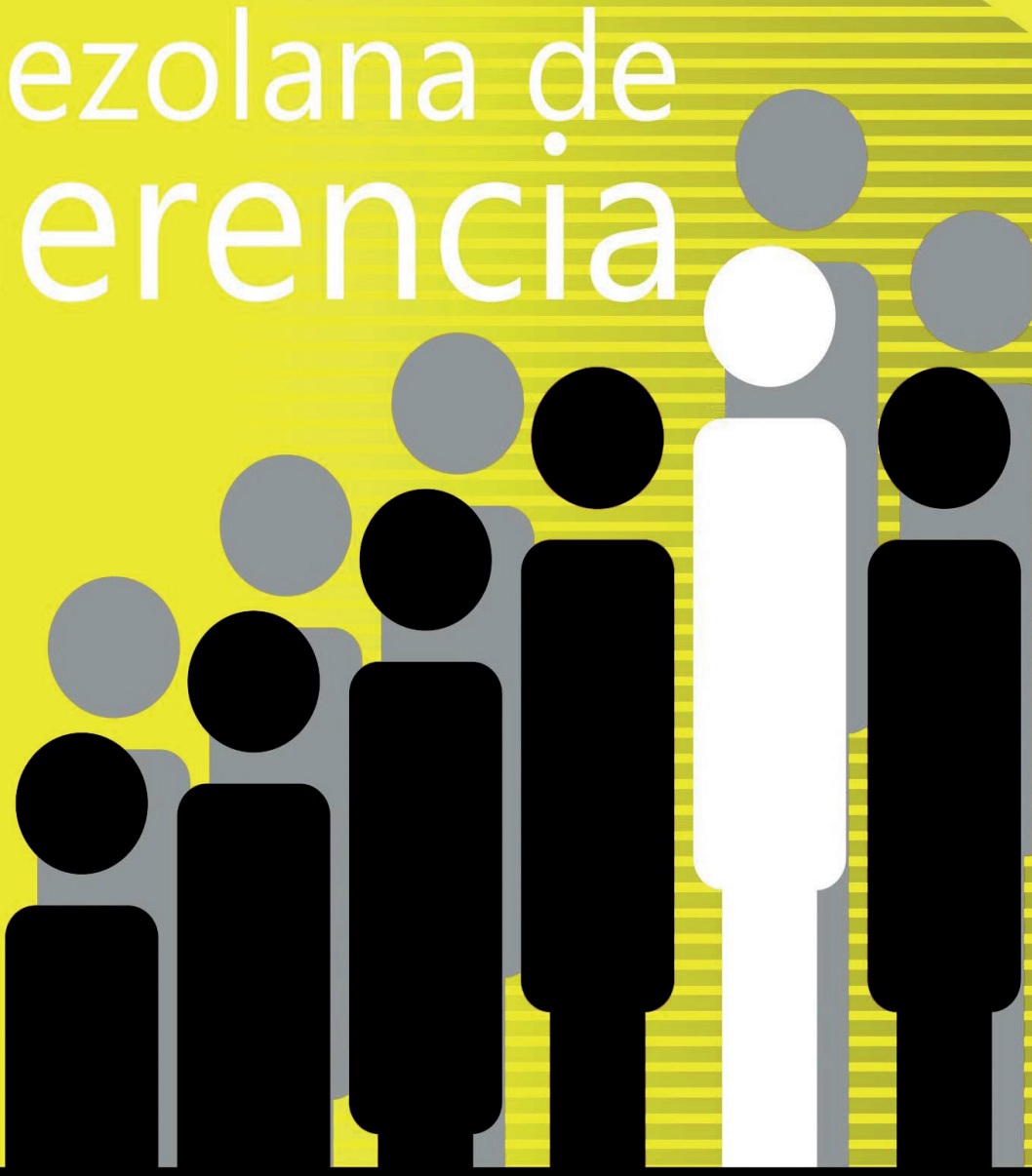


\title{
E-Gobierno local en México en tiempos de Covid-19
}

\author{
Carrera Mora, Oscar Yahevh* \\ Villafuerte Valdés, Luis Fernando* \\ Romero León, Daniel Arturo ${ }^{* \star *}$ \\ Reyes Mendoza, Selene ${ }^{* * * *}$
}

\section{Resumen}

El objetivo del presente estudio fue determinar el efecto de los factores suficiencia y eficiencia de los sitios web de e-servicios a nivel municipal (local) en la intención de uso por parte de los ciudadanos. Para comprobar la hipótesis se aplicó una encuesta con 12 reactivos divida en tres bloques con preguntas diseñadas a través de una escala Likert de 5 grados, el análisis de datos se aplicó utilizando un análisis descriptivo, de regresión lineal y correlación de Pearson. El instrumento se aplicó a ciudadanos del Estado de Veracruz, México durante los inicios de la pandemia COVID-19. Los resultados indican que existe una correlación positiva entre los factores suficiencia y eficiencia con la intención de uso, permitiendo señalar que la perspectiva del ciudadano se ve motivada por la suficiencia y la eficiencia que le representan los sitios web de e-gobierno.

Palabras clave: Eficiencia; e-servicios; e-gobierno; intención de uso; suficiencia.

* Ph. D. en Ciencias Administrativas y Gestión para el Desarrollo. Profesor de Tiempo Completo, Facultad de Negocios y Tecnologías, Campus Ixtaczoquitlán, Universidad Veracruzana, México. Miembro del Sistema Nacional de Investigadores (SNI). E-mail: ocarrera@uv.mx ORCID: https://orcid.org/0000-0002-9648-5887 (Autor de correspondencia).

** Ph. D. en Ciencias Políticas Profesor de Tiempo Completo, Facultad de Ciencias Administrativas y Sociales, Campus Xalapa, Universidad Veracruzana, México. Cuerpo académico Transformaciones Jurídicas. Miembro del Sistema Nacional de Investigadores (SNI). E-mail: Ivillafuerte@uv.mx ORCID: https://orcid.org/00000001-5555-632X

*** Pd. D. en Relaciones Internacionales e Integración Económica. Investigador de Tiempo Completo, Instituto de Investigaciones y Estudios Superiores Económico-Sociales, Universidad Veracruzana. E-mail: daromero@uv.mx ORCID: https://orcid.org/0000-0003-4934-2477

**** Licenciada en Contaduría y Estudiante de la Maestría en Ingeniería Administrativa. Instituto Tecnológico de Orizaba, México. E-mail: dbpselene@hotmail.com ORCID: https://orcid.org/0000-0001-6214-5051 


\title{
Local e-government in mexico in times of covid-19
}

\begin{abstract}
The objective of this study was to determine the effect of the factors sufficiency and efficiency of e-services websites at the municipal (local) level on the intention of use by citizens. To test the hypothesis, a survey was applied with 12 items divided into three blocks with questions designed through a 5-degree Likert scale; the data analysis was applied using a descriptive analysis, linear regression and Pearson correlation. The instrument was applied to citizens of the State of Veracruz, Mexico during the beginning of the COVID-19 pandemic. The results indicate that there is a positive correlation between the sufficiency and efficiency factors and the intention of use, indicating that the citizen's perspective is motivated by the sufficiency and efficiency of e-government websites.
\end{abstract}

Keywords: Efficiency; e-services; e-government; intention to use; sufficiency

\section{Introducción}

La pandemia del COVID-19 puso de manifiesto la necesidad que tiene la administración pública de contar con mecanismos para otorgar servicios públicos a distancia que favorecieran el distanciamiento social y la no movilidad de la ciudadanía, por lo cual, la aplicación de mecanismos electrónicos como los servicios de e-gobierno son fundamentales en este contexto, sin embargo, existen diversos motivos por los cuales su uso es limitado a pesar de su implementación a nivel local.

De hecho, uno de los de los principales argumentos que han proporcionado las teorías en materia de uso de la tecnológica en la última década (Venkatesh y Bala, 2008), han sido factores como la utilidad percibida y la facilidad de uso (de acuerdo con el Modelo de Aceptación Tecnológica - TAM de Venkatesh y Davis, 2000), por lo que en este momento la utilidad percibida es clave, porque queda más que claro que la implementación de servicios a través de Internet no solo son indispensables, sino también deben ser útiles para la ciudadanía, porque el uso de plataformas de e-gobierno en la actualidad favorece el distanciamiento social.

De tal forma que, este contexto coyuntural ha puesto de manifiesto la importancia que tiene el uso de estos e-servicios, por lo cual, en este estudio se analiza la suficiencia y la eficiencia de dichos servicios proporcionados a través de una plataforma de Internet (e-gobierno), ya que en primera instancia es imprescindible analizar sí el número 
de servicios de e-gobierno ofrecidos a la ciudadanía aunado a una interfaz clara que facilite la interacción con las plataformas infieren en una mejor perspectiva que favorezca el uso de éstas, lo cual pudiera dar como resultado en un mejor aprovechamiento de la tecnología que, a su vez, representará mayor inclusión al e-gobierno en países en desarrollo como México.

Por lo tanto, esta investigación busca determinar el efecto de la suficiencia y la eficiencia de los e-servicios en su intención de uso por parte del ciudadano, haciendo en un primer acercamiento un análisis de los factores a través del modelo del Modelo de Aceptación Tecnológica (TAM), en donde, se establece la hipótesis que indica que la falta de atención a los factores de suficiencia y eficiencia afecta negativamente la intención de uso de los e-servicios del gobierno por parte de la ciudadanía. Para la comprobación de la hipótesis se desarrolló y aplicó una encuesta diseñada a través de una escala Likert de 5 grados que permite conocer la usabilidad de esos servicios y los motivos por los cuales no se utilizan.

\section{2. e-gobierno: revisión de la literatura e importancia}

En una circunstancia inesperada, como la pandemia COVID-19, la prestación de servicios públicos no puede ser suspendida, sino que debe ser entregada a través de diversos medios que garanticen el distanciamiento social, como los son los servicios online utilizando la tecnología digital disponible, de allí la importancia del e-gobierno. Éste es definido de diversas formas, sin embargo, se puede entender al e-gobierno como la utilización por el gobierno de las tecnologías de información y comunicación (TIC) para ofrecer a los ciudadanos la oportunidad de interactuar y realizar operaciones con el gobierno mediante el uso de diferentes medios electrónicos, como lo son: el teléfono, el fax, los quioscos de autoservicio, el correo electrónico/ Internet, entre otros.

En ese sentido, hay una clasificación de diferentes formas de e-gobierno como lo es e-administración, e-políticas, e-democracia, e-servicios, entre otros, este estudio se basó únicamente en el análisis de los e-servicios, éstos comprenden los servicios públicos del gobierno que se pueden ofrecer y realizar a través de una plataforma o sitio web por Internet y se puede acceder a ellos con una computadora, un Smartphone, una tablet, o cualquier dispositivo electrónico y digital con conexión a Internet. Así mismo, estos servicios de e-gobierno se pueden clasificar de acuerdo con la perspectiva de Shareef, et al, (2010), en tres niveles, el primero, el de información o presencia, el segundo, el de interacción y, tercero el transaccional; para esta investigación es fundamental el tercer nivel, ya que implica que el ciudadano puede realizar operaciones sin necesidad de acudir a una oficina de gobierno, lo cual incluye la operación del servicio, la atención, hasta el pago del mismo servicio.

En concreto los e-servicios, se han utilizado ampliamente para llegar a una porción más amplia de la población (Agostino, Arnaboldi, Diaz, 2020, Gozgor, 2020) en la última década y principalmente en este año, ya que en tiempos de crisis lo digital no sólo es conveniente, sino que es un facilitador necesario para que las sociedades funcionen, ya que sostiene las actividades sociales y económicas 
(Taddeo, 2020).

Sin embargo, de acuerdo con diversas investigaciones (ElKheshin, et al, 2020; Kofi, 2017; Xie, Song, Peng y Shabbir, 2016; Soneka, et al, 2020) se ha podido demostrar que la ciudadanía utiliza la tecnología solo sí su percepción en cuanto a diferentes factores es positiva, entre algunos de los factores que se han logrado identificar se encuentran que las plataformas web sean fáciles de usar, funcionales, seguras, confiables, también existe evidencia de la influencia de la edad, de las habilidades tecnológicas y hasta el conocimiento para el uso de la tecnología, entre otros factores más que favorecen en la toma de decisiones del ciudadano en pro de sus intereses, así que dependiendo de cada individuo es como varían los factores que afectan el uso de una tecnología.

La consideración de esos factores que promueven el uso de la tecnología, toma vital importancia para favorecer el distanciamiento social que se ha derivado de la pandemia por COVID-19, por lo cual la administración pública de muchos países ha tenido que buscar alternativas para hacer prevalecer mecanismos para incentivar la distancia social, por lo cual el e-gobierno es un mecanismo lógico de aplicación tras este evento coyuntural. Sin embargo, los estudios demuestran que el número de servicios públicos proporcionados a través de plataformas web, los denominados e-servicios ${ }^{1}$, en las localidades de países en desarrollo aún son limitados o escasos, así mismo, su nivel de madurez que represente una funcionalidad que permita actividades transaccionales no es aún adecuada ni eficiente.

De acuerdo con la perspectiva del e-Government Development Index, cuando los servicios electrónicos son escasos se puede considerar que su e-gobierno es deficiente e insuficiente. Por lo tanto, con esta investigación se busca comprender que tanto afectan esos elementos, como el número de servicios web y la funcionalidad que ellos presentan en la intención de uso de los e-servicios.

De hecho, estudios anteriores (Agata, 2018; Chan y Almaghalsah, 2020; Elkheshin et al, 2020; Xie, et al, 2017; Soneka y Phiri, 2019) sugieren que la percepción del ciudadano con respecto al e-gobierno es vital para su éxito y da como resultado un mejor comportamiento de uso de éste. Por lo cual, este estudio se basa en los factores del TAM como base de los dos elementos propuestos que se incorporan a este modelo que busca identificar la intención del uso, a los cuales se les ha denominado suficiencia y eficiencia. Es importante destacar que a pesar de parecer dos variables lógicas e implícitas la tecnología se ha hecho instrumental para el gobierno en los últimos años (Carrera, et al, 2019), por lo cual, es importante determinar su relación con la intención de uso, así mismo estos factores no se han agrupado en los modelos conocidos, siendo esta la aportación significativa de este estudio.

1 Los servicios electrónicos o e-servicios consisten en un contenido de servicio digital diseñado para reemplazar la experiencia de servicio tradicional de la oficina de atención al público ofrecida a través de plataformas y/o sitios web. 


\section{Modelo de Aceptación Tecnológica (TAM)}

El TAM en este estudio es fundamental, debido a que permite definir y explicar los elementos que motivan la intención de uso de los portales web de e-gobierno, así como de otras tecnologías (Xie et al, 2016). Es importante precisar que el TAM no es la única teoría que permite explicar el comportamiento de uso de una tecnología, también existen otras como la Teoría Unificada de Aceptación y Uso de Tecnología (unified theory of acceptance and use of technology, UTAUT) de Venkatesh et al, (2003) que presenta nueve factores, el Modelos de Adopción de e-gobierno (E-government Adoption Model - GAM) de Shareef et al, (2010) que es un hibrido entre el TAM y la UTAUT, entre otras más.

EI TAM fue seleccionado debido a la simplicidad que representan sus dos variables que lo conforman como factores de adopción tecnológica, ya que estas proporcionan una explicación concreta, permitiendo relacionar tanto a la facilidad de uso percibida como a la utilidad percibida como impulsores para modificar las variables que en este estudio se han considerado como elementos que intervienen en la intención de uso de una tecnología por parte de un individuo.

Así mismo, el modelo TAM (diagrama 1), sugiere que cuando a los usuarios se les presenta una nueva tecnología, hay una serie de factores que influyen en su decisión de uso. Los factores clave definidos por Davis (1989) que posteriormente fueron re-definidos por Venkatesh y Bola (2008) incluyen dos elementos: 1) Utilidad percibida, definido como la percepción del grado que un sistema en particular mejoraría el rendimiento laboral de una persona al usarlo y, la facilidad de uso percibida, que se entiende como la percepción del grado de esfuerzo que realizará una persona al usar un sistema.

\section{Diagrama 1 \\ Modelo de Aceptación Tecnológica (TAM).}

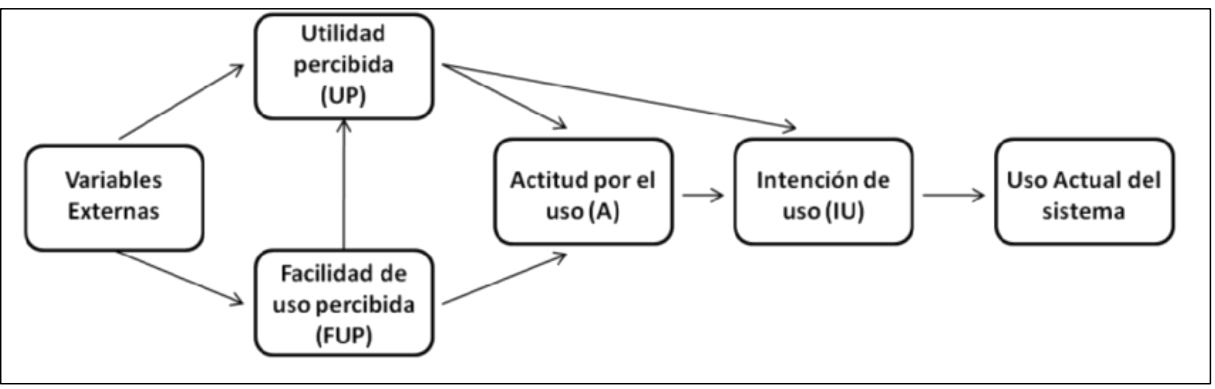

Fuente: Davis et al, (1989). 
De hecho, diversos estudios como los de Carrera, et al, (2019), Elkheshin, et al, (2020), Xie et al, (2017), Soneka y Phiri (2019) (Cuadro 1), entre otros, han utilizado este modelo como elemento explicativo del uso del e-gobierno, en donde, estas dos variables dan forma y/o complementan un sin número de factores más que modifican la intención de uso de las plataformas de e-gobierno bajo condiciones específicas de cada nación, algunos estudios basados en el TAM también incluyen factores de la UTAUT (Teoría unificada de la aceptación y uso de tecnología).

\section{Cuadro 1}

Estudios de e-gobierno basados en el TAM

\begin{tabular}{|c|c|c|c|}
\hline Estudio & Teoría base & Variables & Autores \\
\hline $\begin{array}{l}\text { Assessing the adoption of } \\
\text { e-government using TAM } \\
\text { model: case of Egypt }\end{array}$ & TAM & $\begin{array}{l}\text { - Control del com- } \\
\text { portamiento percibido. } \\
\text { - } \quad \text { Actitud de uso. } \\
\text { ductual de reutilizar. }\end{array}$ & $\begin{array}{l}\text { ElKheshin, Sara Abdelsalam } \\
\text { and Saleeb, Noha (2020). }\end{array}$ \\
\hline $\begin{array}{l}\text { Predictors for e-govern- } \\
\text { ment adoption: integrat- } \\
\text { ing TAM, TPB, trust and } \\
\text { perceived risk. }\end{array}$ & TAM y UTAUT & $\begin{array}{ll}\text { - } & \text { Diseño del sitio } \\
\text { web. } & \text { Nivel educativo. } \\
\text { - } & \text { Confianza en el } \\
\text { - } & \text { Valor público per- } \\
\text { gobierno y en internet. } \\
\text { cibido. }\end{array}$ & $\begin{array}{l}\text { Xie, Q., Song, W., Peng, X., y } \\
\text { Shabbir, M. (2017). }\end{array}$ \\
\hline $\begin{array}{l}\text { A Model for Improving } \\
\text { E-Tax Systems Adoption } \\
\text { in Rural Zambia Based on } \\
\text { the TAM Model }\end{array}$ & TAM & $\begin{array}{l}\text { Utilidad percibida } \\
\text { Facilidad de uso } \\
\text { Riesgo percibido }\end{array}$ & Soneka y Phiri (2019). \\
\hline $\begin{array}{l}\text { Predictors for e-govern- } \\
\text { ment adoption: integrat- } \\
\text { ing TAM, TPB, trust and } \\
\text { perceived risk. }\end{array}$ & TAM y TPB & $\begin{array}{l}\text { Confianza } \\
\text { Riesgo percibido. }\end{array}$ & $\begin{array}{l}\text { Xie, Q., Song, W., Peng, X., y } \\
\text { Shabbir, M. (2016). }\end{array}$ \\
\hline
\end{tabular}

Fuente: Elaboración propia

Así mismo, estos estudios permiten identificar elementos importantes que intervienen en el comportamiento de uso de un individuo en cuanto al e-gobierno se refiere, sin embargo, la mayoría de los estudios analizados centra su atención en una dimensión particular, ya sea en la dimensión de acceso o en la dimensión de uso (Carrera et al, 2019), dicho en otras palabras fijan su atención en la realización de estudios que identifican elementos de la infraestructura o de la usabilidad para confirmar el éxito del e-gobierno y mejorar la usabilidad. 1.1. 


\subsection{Estudios de e-gobierno}

La revisión de la literatura de autores como Byun y Finnie (2010); Carrera, et al, (2019); Xie, et al, (2020); Zárate, et al, (2020) permiten constatar que son pocas las investigaciones que buscan un hibrido de variables entre ambas dimensiones mencionadas anteriormente (dimensión de acceso y de uso), así mismo, diversos autores (Agata, 2018; Chan y Almaghalsah, 2020) afirman que la creación de plataformas o sitios web del gobierno deben ser útiles y funcionales, permitiendo con ello, la plena satisfacción de los beneficios derivados de las nuevas tecnologías, por lo cual, su eficiencia es una condición indispensable para mejorar la intención de uso de estos mecanismos.

Así, el diseño puede afectar el uso de un sitio web cuando se busca la información para realizar el proceso y se muestra en la pantalla, por consiguiente, es importante la eficiencia en la entrega de información en una plataforma web, ya que su funcionalidad puede ser limitada, derivado de un diseño poco lógico para el usuario (Pinandito et al, 2017), por lo cual, se puede apreciar que la intención de uso de un sitio web pudiese ser influida por varios factores (Zárate et al, 2020) que, en este caso representan la percepción que el usuario tendrá de la funcionalidad, misma que radica de una eficiencia positiva o negativa derivada de su funcionalidad. Se entiende por eficiencia en este estudio a la capacidad que tiene un sitio web para realizar o cumplir adecuadamente una función determinada con el menor esfuerzo posible (Li et al, 2009).

Por otra parte, las investigaciones (Hariguna et al, 2017; Nawafleh, 2018) indican que en la actualidad el número y/o cantidad de sitios web de e-gobierno, deben ir día con día en aumento, por lo cual se puede establecer que, sí el ciudadano no encuentra de primera instancia el servicio que requiere su percepción le hará pensar que los demás existentes no le son útiles, en ese sentido, expertos en el ámbito del desarrollo de software y aplicaciones web, explican que en cuanto más servicios estén digitalizados, mayor será el número de individuos que si integren al uso de los servicios de forma digital, ya que se perciben mayores ventajas al existir la integración de la mayoría de servicios a los sitios web (AlcarazQuiles, et al, 2018; Byun y Finnie, 2010; Singh, et al, 2016).

Por lo cual, en esta investigación se proponen dos variables la de suficiencia y la de eficiencia que se podrán sumar a los factores de adopción tecnológica identificados en otros estudios, estos elementos están situados en la dimensión de infraestructura que son derivados de la percepción de la ciudadanía con respecto a las plataformas de e-gobierno, mismos factores que afectan a las variables de comportamiento de uso definidos en el modelo del TAM (Utilidad percibida y facilidad de uso).

Estos dos factores propuestos se destacan como dilemas principales de estudio en la investigación y se pueden comprender de la siguiente manera:

a) La suficiencia del e-gobierno: se puede comprender como el número de servicios públicos otorgados a través de plataformas web de la administración pública y;

b) La eficiencia, como la percepción de funcionalidad que estas plataformas web de e-gobierno presentan.

Hipotéticamente, estos elementos descritos afectan a los factores del TAM, el de suficiencia a la utilidad percibida 
en función de, sí existen los servicios de e-gobierno requeridos $y$, segundo la eficiencia afecta la percepción de facilidad de uso, en función de sí este servicio de e-gobierno es fácil de usar permitiendo realizar o no el trámite para el cual fue diseñada o no, la plataforma. Así, tomando como principio el modelo del TAM, se construyó un modelo basado en los dos factores, en donde, se construyen dos variables adyacentes denominadas suficiencia y eficiencia.

A partir del análisis teórico presentado anteriormente fue posible identificar dos factores que están ausentes de la literatura, como lo son la suficiencia y la eficiencia, estos se contrastan con el modelo del TAM, por lo cual, la definición de la hipótesis que dio origen a este estudio es la siguiente:

$\mathrm{H1}$ : La suficiencia y eficiencia de las plataformas web de servicios de e-gobierno, está relacionada negativamente con la intención de uso de los e-servicios por parte de los ciudadanos (diagrama 2).

H1a: Cuando los servicios de e-gobierno no son suficientes, se genera una percepción de utilidad negativa, disminuyendo la intención de uso de los e-servicios por parte de los ciudadanos.

$\mathrm{H} 1 \mathrm{~b}$ : Cuando los servicios de e-gobierno no son eficientes, se propicia una perspectiva de facilidad de uso negativa, disminuyendo la intención de uso de los e-servicios por parte de los ciudadanos.

\section{Diagrama 2 \\ Hipótesis de la Investigación.}

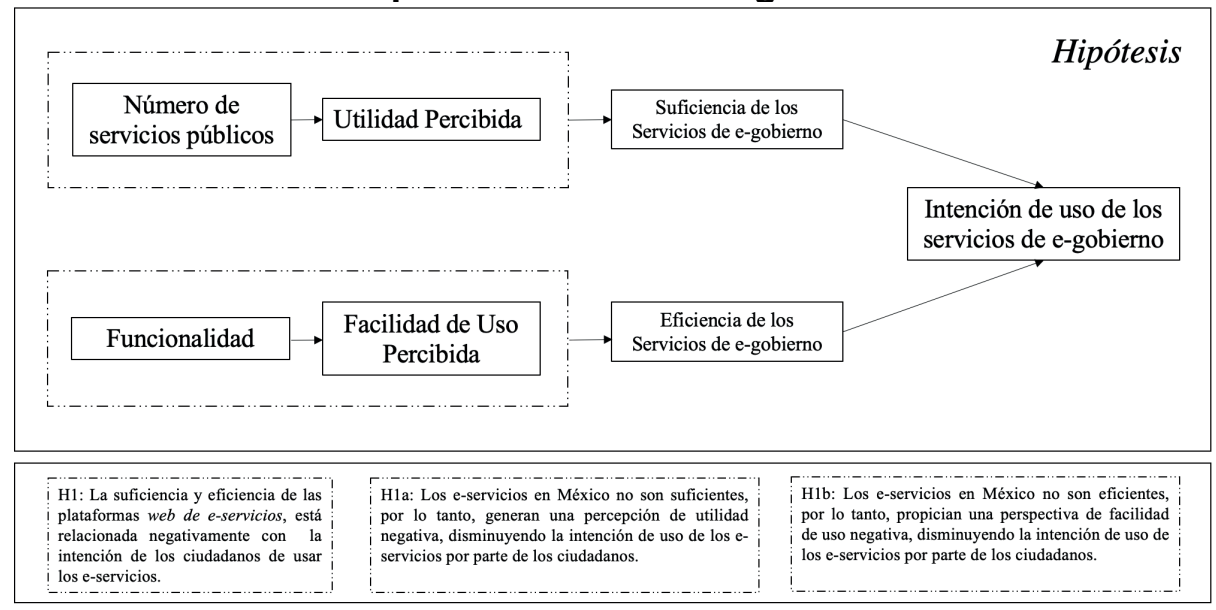

Fuente: Elaboración propia. 


\section{Consideraciones metodológicas de la investigación}

Para la comprobación de la hipótesis se desarrolló un cuestionario con preguntas esbozadas por medio de una escala Likert de cinco grados, en donde, por una parte, se obtuvieron datos generales como: Edad, sexo, nivel de estudios $y$, por la otra parte, se diseñaron tres bloques de cuatro preguntas cada uno para comprobar la hipótesis del estudio planteada002E

$$
\text { México es un país }
$$
demográficamente extenso con una población de 127.09 millones de mexicanos, por lo cual se tomó al Estado de Veracruz como un estudio de caso. Una de las motivaciones principales para tomar este Estado, es que es una de las entidades con el menor desarrollo e implementación de servicios de e-gobierno con un $34.44 \%$ con respecto al más alto que cuenta con un $55.11 \%$ de los servicios reportado por los estudios del U-GOB (2020), así mismo, la usabilidad de sus e-servicios se encuentran por debajo de la media.

Los e-servicios a nivel local (municipal) que se ofrecen de manera transaccional en los municipios que conforman el Estado de Veracruz son en promedio tres, los cuales consisten: pago del impuesto predial, pago de servicios de recolección de basura y uso de suelo, por lo tanto, estos son los servicios municipales que fueron evaluados en cuanto a funcionalidad y usabilidad, además, durante la pandemia se ha seguido ofreciendo estos servicios in situ, lo cual permite la elección del ciudadano para utilizar o no los sitios web.

Una exploración de los sitios web de e-gobierno de los 212 municipios que conforman al Estado de Veracruz, permitió identificar que sólo siete de ellos cuentan con plataformas en el nivel transaccional, los municipios que cuentan con algún e-servicio son: Boca del Río, Córdoba, Coatzacoalcos, Orizaba, Poza Rica, Xalapa y Veracruz, por lo cual, se aplicó las encuestas a ciudadanos de estos municipios, verificando a través del campo edad, que cada uno de los encuestados contara con una edad superior a las 18 años para poder realizar estos trámites.

Así mismo, se diseñó una pregunta de control, a través de la cual se buscó evaluar únicamente a aquellos ciudadanos que hubieran hecho supieran de la existencia de los e-servicios, así como, aquellos que lo intentaron utilizar pero que no lo han hecho por distintos motivos debido a que el servicio no se encontraba disponible. La pregunta fue la siguiente: ¿Sabes que existen sitios web para realizar el pago de servicios del gobierno?, todas aquellas respuestas que fueron resultas con un No, se eliminaron de la muestra de análisis. Por lo cual, las 670 respuestas analizadas únicamente son de aquellos individuos que pasaron el primer filtro.

La encuesta fue puesta on-line, con lo cual se aseguró que los individuos que contestaron el instrumento tienen el conocimiento del uso de este tipo de tecnología y las habilidades digitales para dar una respuesta objetiva al respecto. El instrumento de recolección de datos se diseñó en la plataforma de Microsoft Forms, con lo cual se compartió el enlace de acceso a las encuestas por medio de WhatsApp, Facebook y canales institucionales de la Universidad Veracruzana, como el correo electrónico, durante un periodo de cuatro meses que corresponden 
del 01 de mayo al 01 de septiembre del año 2020, pudiendo recolectar 670 respuestas que permiten el análisis respectivo para la comprobación de la hipótesis.

\section{Suficiencia y eficiencia de las plataformas web de servicios de e-gobierno: resultados}

El análisis de los datos se divide en dos apartados, el primero, consta de un análisis descriptivo de los datos obtenidos en la primera sección del cuestionario $y$, el segundo, un análisis de regresión lineal y otro correlacional que permiten verificar la hipótesis de la investigación.

\subsection{Análisis descriptivo}

El análisis permitió identificar a través que el $71 \%$ de la población encuestada ha realizado algún trámite de gobierno empleando Internet, por lo cual los resultados indican que 479 usuarios si han ocupado el e-gobierno para realizar trámites, gráfico 1 .

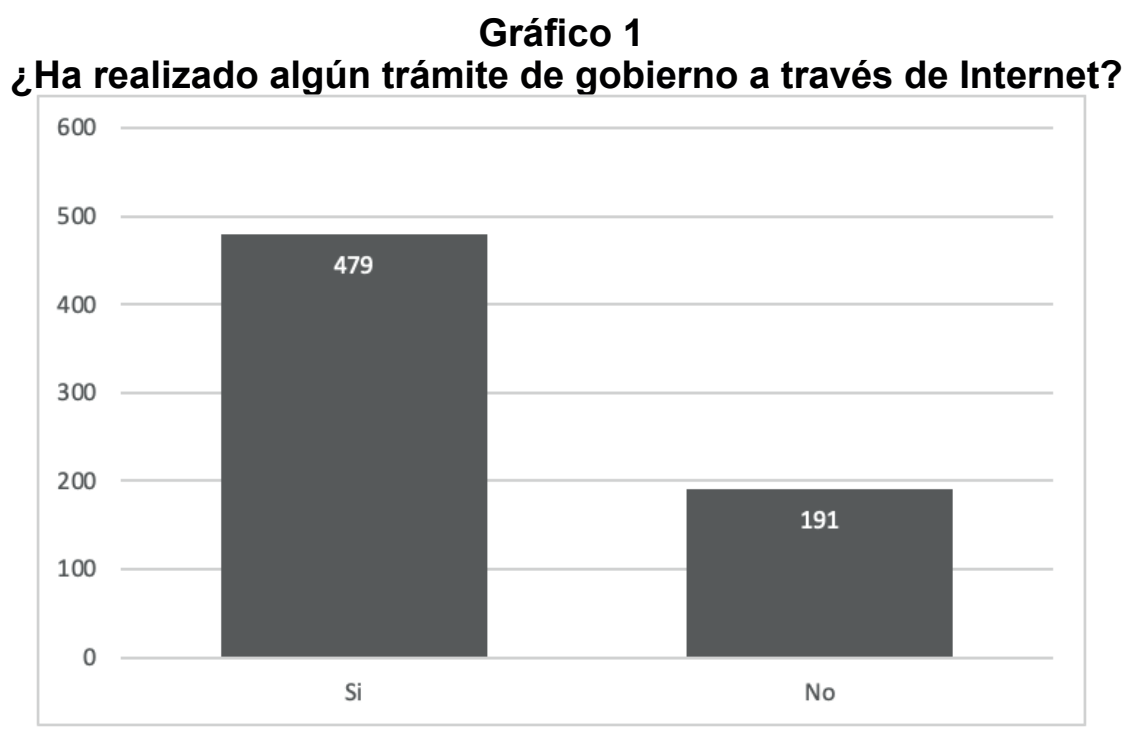

Fuente: Elaboración propia.

Sin embargo, del porcentaje restante $(29 \%)$ se puedo observar que aquellos individuos que no han hecho uso alguna vez de un servicio de e-gobierno son aún una cantidad amplia, siendo 105 ciudadanos quienes manifiestan que el motivo por el cual no lo han utilizado es porque no existe el e-servicio, distinguiéndose la insuficiencia de los servicios (Gráfico 2). 
Carrera Mora, Oscar Yahevh; Villafuerte Valdés, Luis Fernando; Romero León Daniel

Arturo; Reyes Mendoza Selene

E-Gobierno local en México en tiempos de Covid-19

Gráfico 2

¿Cuál ha sido el motivo por el cual no ha utilizado un sitio web de e-gobierno?

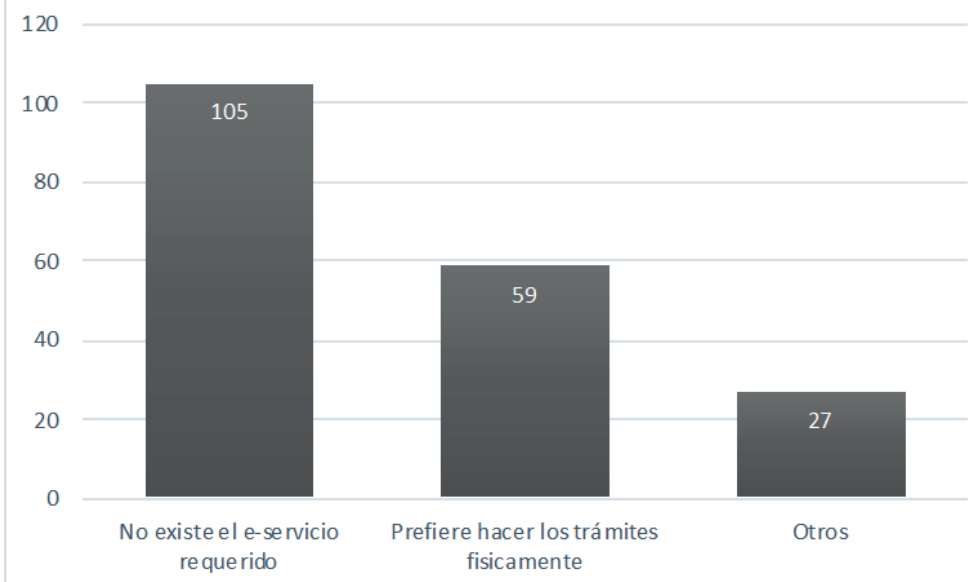

Fuente: Elaboración propia.

Por otra parte, se analizó el uso que se le dio a los servicios públicos tradicionales para poderlos contrastar contra los e-servicios $y$, durante el periodo que se analizó en el cual transcurre la pandemia de COVID 19, del total de la población encuestada de 670 personas, solo el $37 \%$ que corresponde a 248 ciudadanos, si utilizó los servicios públicos (gráfico 3).

\section{Gráfico 3 \\ ¿Realizó algún trámite ante el gobierno municipal durante la pandemia COVID-19?}

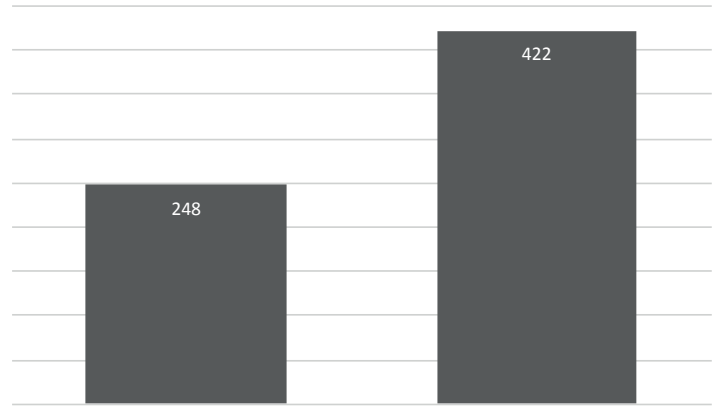

Fuente: Elaboración propia. 
De lo anterior, se puede observar en el Gráfico 4, que de la población que requirió realizar algún trámite ante una dependencia gubernamental, únicamente el $45 \%$ de la población encuestada hizo uso de un servicio público de e-gobierno, el resto de la población que equivale al $55 \%$ prefirió asistir directamente a la oficina de gobierno a pesar de los requerimientos de distanciamiento social.

\section{Gráfico 4 \\ ¿Cuál fue el medio a través del cual realizó su trámite?}

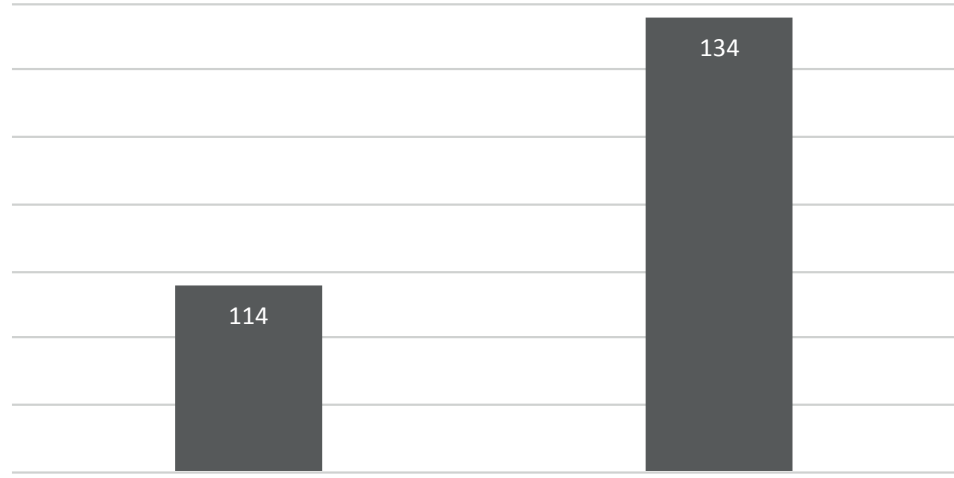

Fuente: Elaboración propia

El Gráfico 5 permite observar que de esa población que decidió asistir a realizar su trámite a la oficina gubernamental (ventanilla), fue motivado principalmente por no existir el servicio público digitalizado, dato que equivale al $45 \%$ de la población que realizó un trámite en ventanilla, lo cual en este estudio se le conoce como la suficiencia y el $17 \%$ lo hizo por considerar que las plataformas de e-gobierno no son funcionales, lo que en este estudio se le conoce como eficiencia. 


\section{Gráfico 5 \\ Motivo por el cual no realizó el trámite a través de Internet}

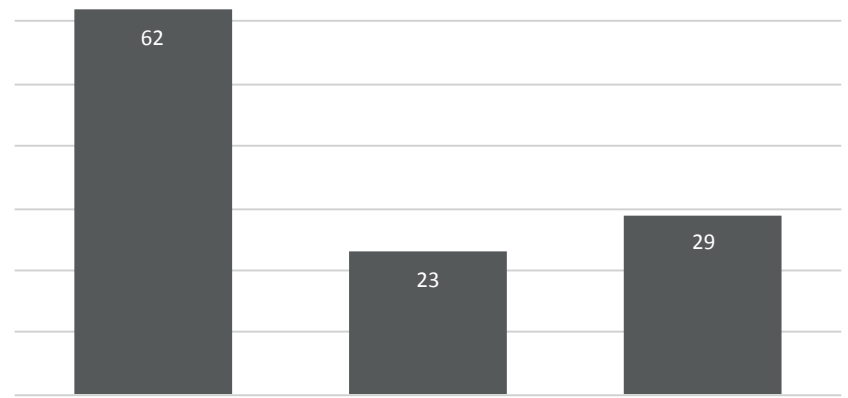

Fuente: Elaboración propia.

\subsection{Análisis de fiabilidad del instrumento (Alfa de Cronbach)}

Se realizó un análisis de fiabilidad del instrumento a través del método de Alfa de Cronbach. El resultado obtenido de fiabilidad del software IBM SPSS 25 , presenta un coeficiente alfa $>.8$, por lo cual es bueno de acuerdo con la perspectiva de Frias-Navarro (2019), ya que el resultado es de .0808 (tabla 1), corroborando la fiabilidad de los ítems del instrumento y su correlación entre ellos.

Tabla 1

\section{Fiabilidad del instrumento}

\begin{tabular}{cc}
\hline \multicolumn{2}{c}{ Estadisticas de fiabilidad } \\
\hline Alfa de Cronbach & $\mathrm{N}$ de elementos \\
\hline .808 & 12 \\
\hline
\end{tabular}

Fuente: Elaboración propia.

\subsection{Análisis de regresión lineal}

Se validó el grado de asociación de los factores suficiencia y eficiencia definidos en las hipótesis $\mathrm{H} 1$ (a) y $\mathrm{H} 1$ (b), determinando que la muestra es válida, y es aceptable basándonos en el nivel de confianza definido del 95\%. Asimismo, se identificó un coeficiente de correlación múltiple del 0.543, indicando una correlación positiva fuerte y un coeficiente de determinación de $\mathrm{R}$ cuadrado de 0.293 , con un error estándar de la estimación de .60164 para una muestra de 670 individuos (tablas 2 y 3). De este modo, se demuestra que los factores de adopción suficiencia y eficiencia tienen un efecto en la intención de uso de la ciudadanía de un e-servicio. 
Tabla 2

Resultado del análisis de regresión lineal (a).

\begin{tabular}{ccccc}
\hline \multicolumn{5}{c}{ Resumen del modelo } \\
\hline Modelo & $\mathrm{R}$ & R cuadrado & R cuadrado ajustado & Error estándar de la estimación \\
\hline 1 & $.543^{\text {a }}$ & .295 & .293 & .60164 \\
\hline \multicolumn{4}{c}{ a. Predictores: (Constante), EFICIENCIA, SUFICIENCIA } \\
\hline
\end{tabular}

Fuente: Elaboración propia.

Tabla 3

Resultado del análisis de regresión lineal (b).

\begin{tabular}{|c|c|c|c|c|c|c|}
\hline \multicolumn{7}{|c|}{ Coeficientes $^{a}$} \\
\hline & \multirow{2}{*}{$\begin{array}{l}\text { Modelo } \\
\text { B }\end{array}$} & \multicolumn{2}{|c|}{ Coeficientes no estandarizados } & \multirow{2}{*}{$\begin{array}{c}\text { Coeficientes } \\
\text { estandarizados }\end{array}$} & \multirow{2}{*}{$\mathrm{t}$} & \multirow{2}{*}{ Sig. } \\
\hline & & Desv. Error & Beta & & & \\
\hline \multirow{3}{*}{1} & (Constante) & 1.746 & .146 & & 11.963 & .000 \\
\hline & SUFICIENCIA & .087 & .029 & .098 & 2.975 & .003 \\
\hline & EFICIENCIA & .526 & .033 & .522 & 15.900 & .000 \\
\hline
\end{tabular}

a. Variable dependiente: INTENCIÓN-USO

Fuente: Elaboración propia.

\subsection{Análisis de correlación de Pearson}

El coeficiente de correlación puede tomar un rango de valores de +1 a -1 . Un valor de 0 indica que no hay asociación entre las dos variables. Un valor mayor que 0 indica una asociación positiva. Es decir, a medida que aumenta el valor de una variable, también lo hace el valor de la otra. Un valor menor que 0 indica una asociación negativa; es decir, a medida que aumenta el valor de una variable, el valor de la otra disminuye.

En ese sentido, este análisis nos permite evidenciar la asociación que existe entre cada una de las variables propuestas con la intención de uso, en donde la asociación de la eficiencia es de .535 siendo positiva, con respecto a la suficiencia el grado de asociación es de .167 y al ser superior a 0 también es positivo (tabla 4) 
Carrera Mora, Oscar Yahevh; Villafuerte Valdés, Luis Fernando; Romero León Daniel

Arturo; Reyes Mendoza Selene

E-Gobierno local en México en tiempos de Covid-19

Tabla 4

Resultados del análisis de correlación de Pearson

\begin{tabular}{ccccc}
\hline \multicolumn{5}{c}{ Correlaciones } \\
\hline & INTENCIÓN-USO & EFICIENCIA & SUFICIENCIA \\
\hline \multirow{3}{*}{ INTENCIÓN-USO } & $\begin{array}{c}\text { Correlación de } \\
\text { Pearson }\end{array}$ & 1 & $.535^{* *}$ & $.167^{* *}$ \\
\cline { 2 - 5 } & Sig. (bilateral) & & .000 & .000 \\
\cline { 2 - 6 } & $\mathrm{N}$ & 670 & 670 & 670 \\
\hline
\end{tabular}

**. La correlación es significativa en el nivel 0,01 (bilateral).

Fuente: Elaboración propia.

Con base en los datos mostrados es posible observar en el diagrama 2 , el esquema de la hipótesis con el dato del coeficiente de relación positivo de ambos factores propuestos y el coeficiente de correlación para cado uno de los factores de forma individual, lo cual permite dar evidencia de la comprobación de la hipótesis.

\section{Diagrama 2 \\ Correlación de los factores con la intención de uso de los e-servicios}

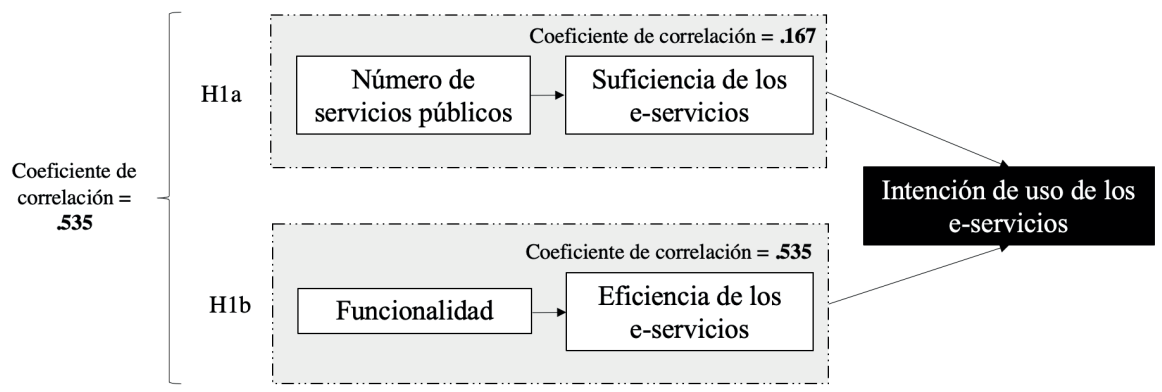

Fuente: Elaboración propia.

Así el supuesto que se ha comprobado sugiere que tanto la suficiencia como la eficiencia de los e-servicios si influyen en la intención de uso de los servicios de e-gobierno y a pesar de existir algunos servicios que ya operan a través de Internet, los individuos relacionan la cantidad de servicios con la eficiencia de éste, modificando el comportamiento de uso de estos mecanismos, lo cual, genera la necesidad para la administración pública 
de poner atención en estos y otros factores para mejorar la usabilidad e las plataformas de e-gobierno.

\section{Conclusiones}

\begin{abstract}
Por lo tanto, es posible concluir que estos factores identificados influyen en el número de usuarios, limitando la eficiencia económica de la administración pública, debido a que aún es necesario invertir grandes recursos en los procesos manuales que no han podido ser sustituidos por los procesos automatizados que ofrecen los e-servicios, así mismo, no se favorece el distanciamiento social con la aplicación de estos e-servicios.
\end{abstract}

Lo anterior, genera diferentes efectos negativos, en primer lugar, la falta de inclusión de la ciudadanía a estos servicios, segundo, no se favorece al distanciamiento social requerido, obligando al ciudadano a desplazarse para realizar sus trámites presencialmente, esto lleva a un tercer ámbito, no se cumple con los objetivos del e-gobierno planteados por los organismos internacionales y la gobernanza en cuanto a la transparencia en los procesos y la disminución de la corrupción en la realización de trámites gubernamentales y, cuarto, la falta de automatización de los procesos limita la concentración de información para un procesamiento de datos que pudiera favorecer en una toma de decisiones más centrada, consciente y eficiente, porque al limitar el número de e-servicios, la información existente en tiempo real que pudiese utilizar el tomador de decisiones de la administración pública está limitada por la falta de bases de datos que le permitan filtrar información de forma eficaz y eficiente.
Por otra parte, hay otro pendiente muy importante en el desarrollo de este ensayo, y este es la infraestructura tecnológica para el desarrollo de la eficiencia del e-gobierno, a la cual se requiere hacer énfasis especialmente en la implementación de banda ancha y la dotación de una mayor capacidad del Internet en mayores sectores sociales vulnerables en el país, lo cual permita aumentar el número de usuarios de los sitios web de e-gobierno, el cual es indispensable para mantener, en primer lugar, los paradigmas para los cuales fue creado el e-e-gobierno, entendiéndose como lograr la eficiencia y la eficacia de la administración pública y, segundo, en este proceso de distanciamiento social obligado, es indispensable promover este tipo de plataformas, favoreciendo la inclusión de la ciudadanía a través de la utilización de estas herramientas.

Así mismo, se puede evidenciar que el ciudadano espera la incorporación de mayores plataformas web que ofrezcan servicios públicos de e-gobierno, ya que el alejamiento de estos e-servicios, no solo radica en el desconocimiento o la renuencia al cambio, sino en la cantidad tan limitada de e-servicios existentes. Por lo cual, los futuros desarrollos de e-servicios a nivel municipal en países en desarrollo como México deben estar encaminados al cumplimiento de las diversas perspectivas sociales a través de la consideración de estos factores y otros más, que han sido ya detectados en otras investigaciones para la construcción de sus estrategias digitales.

Por último, una línea de investigación que queda abierta, es poder comprobar está misma hipótesis en países que cuenten con un gran número de e-servicios para verificar la suficiencia y por otra parte diseñar un 
Carrera Mora, Oscar Yahevh; Villafuerte Valdés, Luis Fernando; Romero León Daniel

Arturo; Reyes Mendoza Selene

E-Gobierno local en México en tiempos de Covid-19

sitio web con los factores tecnológicos identificados en diversos estudios para verificar la perspectiva de eficiencia con estos factores, ya que la tecnología no es un elemento instrumental que al implementarse garantiza su utilización, ésta requiere de cumplir con diferentes perspectivas y particularidades de los usuarios, siguiendo las perspectivas de la gobernanza y del buen vivir que buscan la inclusión de la ciudadanía en todos los medios posibles para asegurar la participación.

\section{Referencias bibliográficas}

Alcaraz-Quiles F.J., Urquia-Grande E., Muñoz-Colomina C.I., Rautiainen A. (2018) E-Government Implementation: Transparency, Accessibility and Usability of Government Websites. In: Alcaide Muñoz L., Rodríguez Bolívar $M$. (eds) International E-Government Development. Palgrave Macmillan, Cham. https://doi.org/10.1007/978-3319-63284-1 12

Agata, A. (2018). Usability and functionality of websites of commune offices as stimulants of sustainable development of e-government. SHS Web of Conferences, 57, 1-11. $\quad$ https://doi.org/10.1051/ shsconf/20185701029

Agostino, D., Arnaboldi, M., y Diaz, M. (2020). New development: COVID-19 as an accelerator of digital transformation in public service delivery. Public Money \& Management, 1-4. https://doi.org/10. 1080/09540962.2020.1764206

Byun, D-H. y Finnie, G. (2010). Evaluating usability, user satisfaction and intention to revisit for successful e-government websites. Electronic Government, and International Journal, 8(1). https://doi.org/10.1504/

\section{EG.2011.037694}

Chan, C.H., y Almaghalsah, H. (2020). Usability evaluation of e-government websites: A case study from Taiwan. International Journal of Data and Network Science, 4(2), 127-138. https://doi.org/10.5267/j. iidns.2020.2.004

Davis, F.D. (1989). Perceived usefulnes, perceived ease of use, and user acceptance of information technology. MIS Quarterly, 13(3).

ElKheshin, S., y Saleeb, N. (2020). Assessing the adoption of e-government using TAM model: case of Egypt. International Journal of Managing Information Technology, 12(1), 1-14. https://doi.org/10.5121/ ijmit.2020.12101

Frias-Navarro, D. (2019). Apuntes de consistencia interna de las puntuaciones de un instrumento de medida. Universidad de Valencia. España. https://www.uv.es/friasnav/ AlfaCronbach.pdf

Gozgor, G. (2020). Global Evidence on the Determinants of Public Trust in Governments during the COVID-19. Cesifo Working Papers, 1(8313), 1-19. https://ssrn.com/ abstract $=3618837$

Hariguna, T., Lai, M., Hung, C.W., y Chen, S.C. (2017). Understanding information system quality on public e-government service intention: an empirical study. International Journal of Innovation and Sustainable Development, 11(2-3). https://doi. org/10.1504/IJISD.2017.083290

Kofi, I. (2017). Citizens' Readiness to Adopt and Use E-government Services in the City of Harbin, China. International Journal of Public Administration, 41(4), 297-307. https://doi.org/10.1080/01900692.20 16.1263658 
Li, Z., Li, J., y Li, X. (2009). A Study on Website Operation Efficiency Evaluation: Based on DEA Model. International Conference on Management and Service Science, Wuhan, 1-6, https://doi.org/10.1109/ ICMSS.2009.5301119

Nawafleh, S. (2018). Factors affecting the continued use of e-government websites by citizens: An exploratory study in the Jordanian public sector. Transforming Government: People, Process and Policy, 12(3/4), 244264. https://doi.org/10.1108/TG-022018-0015

Pinandito, A., Az-zahra, H. M., Fanani, L., y Putri, A. V. (2017). Analysis of web content delivery effectiveness and efficiency in responsive web design using material design guidelines and User Centered Design. 2017 International Conference on Sustainable Information Engineering and Technology (SIET), Malang, 435-441, https://doi.org/10.1109/ SIET.2017.8304178.

Shareef, M.A., Kumar, V., Kumar, U. y Dwivedi, Y. K. (2010). e-Government Adoption Model (GAM): Differing service maturity levels. Government Information Quarterly, 28, 17-35.

Soneka, P.N. and Phiri, J. (2019) A Model for Improving E-Tax Systems Adoption in Rural Zambia Based on the TAM Model. Open Journal of Business and Management, 7, 908-918. https://doi.org/10.4236/ ojbm.2019.72062

Singh, T., Malik, S., y. Sarkar, D. (2016). E-commerce website quality assessment based on usability, 2016 International Conference on Computing, Communication and Automation (ICCCA). Noida,
101-105. https://doi.org/10.1109/ CCAA.2016.7813698.

Taddeo, M. (2020). The Ethical Governance of the Digital During and After the COVID $\square 19$ Pandemic. Minds and Machines Springer, 1-6. https://doi.org/10.1007/s11023-02009528-5

U-GOB (2018). Ranking de Portales de Gobierno Estatal 2018. https://ugob.com/ranking-de-portales-degobierno-estatal-2018/

Venkatesh, V., y Bola, H. (2008). Technology acceptance model 3 and a research agenda on interventions, Decision Sciences, 39 (2), 273-315.

Venkatesh, V., y Davis, F. (2000). A theoretical extension of the technology acceptance model: four longitudinal field studies, Management Science, 46 (2), 186-204.

Venkatesh, V., Morris, M., Davis, B., y Davis, F. (2003). User Acceptance of Information Technology: Toward a Unified View. MIS Quarterly, 27(3), 425-478.

Xie, Q., Song, W., Peng, X., y Shabbir, M. (2017). Predictors for e-government adoption: integrating TAM, TPB, trust and perceived risk. The Electronic Library, 35(1), 2-20. https://doi. org/10.1108/EL-08-2015-0141

Zárate J.A., Rosales, A., Guerrero M.G., Ríos, B.O., y Rivera, P. (2020). Measuring the facility of use of a website designed with a methodology based on concepts of design of ontologies. International Journal of Innovation Engineering and Science Research, 2(4), 33-41. http://www. ijiesr.com/liebrary/e15/9114259909. pdf 